\title{
Example of Experimental Use of 3D Measurement System for Construction Robot Based on Component Design Concept
}

\author{
Hiroshi Yamamoto \\ Research Coodinator \\ Public Works Research Inst. \\ Tukuba-si Ibaraki Pref. Japan
}

\author{
Ken Endo \\ Research Depertment \\ Tokyu Construction Co.,Ltd \\ Sagamihara-si kanagawa \\ Pref. Japan
}

\author{
Yutaka Ishimatsu \\ Senior Researcher \\ Public Works Research Inst \\ Tukuba-si Ibaraki Pref. Japan
}

\author{
Minoru Masuda \\ Mamager \\ Toa Corporation \\ Chiyoda-ku Tokyo Japan
}

\author{
Syuji Ageishi \\ Vice Manager \\ Japan Construction Method \\ and Machinery R .I. \\ Fuji-si Sizuoka Pref. Japan \\ Masayuki Uchida \\ System Sales engineer \\ GNSS Technologies Co.,Ltd \\ Shinjiku-ku Tokyo Japan
}

\author{
Naohiro Ikeda \\ Senior Researcher \\ Japan Construction Method \\ and Machinery R .I. \\ Fuji-si Sizuoka Pref. Japan
Hiroyoshi Yamaguchi Manager
Komatsu Engineering Corp.
Hiratsuka-si kanagawa Pref. Japan

Abstract: In civil engineering projects, operators run their machine, while monitoring machine operation data, soil conditions, situation around their machines, and so on. They basically follow the work directions that are issued based on the machine design and field conditions, such as topography of the site. An increasing number of construction robots are used in restoration work in disaster-stricken areas, and the advent of robots that can effectively utilize design data, field topographical conditions and such in response to the operator's demand is hoped for.

This paper presents information about installation and demonstration tests of the measurement support system that has been designed with the "component design" concept in mind. The 3D measurement system for construction site is composed of three components and it is a part of the General Technology Development Projects called "Development of IT-Assisted System Based on Robots, etc.” of the Ministry of Land, Infrastructure and Transport. Based on this concept, the system configuration could significantly be made flexible, thereby facilitating reuse of this support system on a component-by-component basis.

Keywords: Construction robot, component design, measurement support system, demonstration test

\section{Introduction}

Since the construction robots have to operate in an environment specific to construction, i.e., outdoor site, they have been developed on the basis of the element technologies peculiar to construction robots. In order to secure work safety, remote operation of the robots has become feasible by means of remote control, video application, and communication technologies. As a means for grasping the situation and conditions at the disaster-stricken area, technologies of having mastery of various sensors have been introduced as part of requirements for field data acquisition (typically, volcanic gas density metering and topographic measurement [1]). Construction robots in the future are expected to be more advanced in utilizing the design data and keeping track of the situation around the robot (external sensors) with the help of rapidly progressing information technology (IT). From the viewpoint that the system should be designed to facilitate corrections and to reuse the element technologies for the purpose of building more advanced and sophisticated system, the element design and experimental use of construction robots were made by using a backhoe as the base machine (hereinafter referred to as the backhoe robot). In this way, the component design and its filed trial were attempted.

\section{Construction Robot System to Be Configured}

\subsection{System configuration requirements}

Construction robots in the future will have subsystems and functions that will make use of large amounts of information, whereas most of conventional construction robots are manufactured only for certain specific applications. Thus, it is predicted that a relatively large and complex system will be required and that the following will be in need in configuring the future system:

(1) To reuse element technologies;

(2) To have flexibility to facilitate modifications of the system; and

(3) To upgrade the usage of information. In building the construction robot, the concept of a component design [2] has been introduced along with development of each element technology.

\subsection{Functional requirements as to construction} With introduction of new IT technology, it becomes possible to upgrade the usage of information and the following new functions are requested of the construction robot:

(1) Utilization of design data

The operator need to know the difference between filed topographical conditions and design conditions in relation to the present position and orientation of the machinery required.

(2) Collection and utilization of topographic features Laser scanner and/or photogrammetry technology can be applied to collection of the topographic features that will be used to support the operator.

(3) Measurement of machine position and orientation The position and orientation of the machine can be known with the GPS receiver, while the correlations 
between the site coordinate system and the machine coordinate system can be understood.

(4) Generation of machine control information Machine control information can be generated by utilizing the design data and field topographical conditions and by grasping the machine position and orientation. At the same time, an interface should be developed to allow optimal corrections and iterations during man-machine coordination.

\section{Component Design for 3D Measurement System for Construction Status}

Measurement system for the construction robot must function such that acquisition of such data as machine position and orientation, topographic data (coordinate data of a group of dots) and video data is synchronously timed and that the work area of these sets of data is converted from the instrument's coordinate systems to the local coordinate system before they are to be delivered in response to the request for measurement from the entire system.

A configuration of this measurement system is given in Figure 3-1.

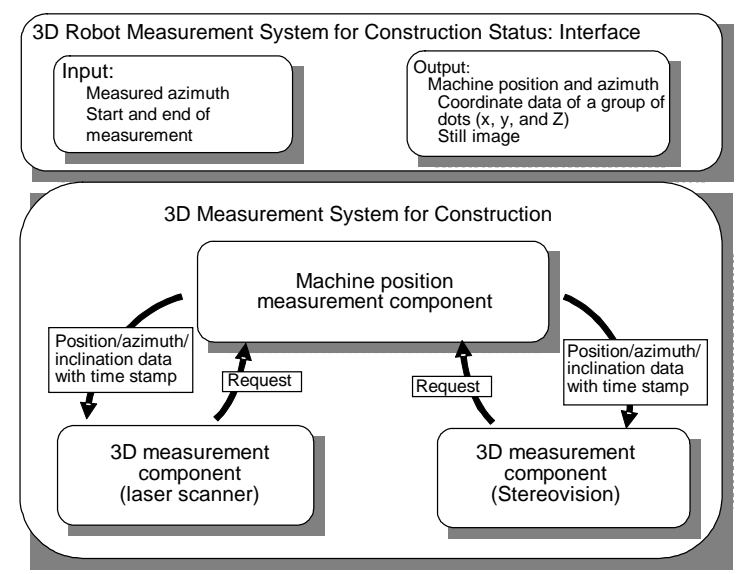

Figure 3-1 Configuration of 3D Measurement System for Construction Status

\section{Experimental Use of Components}

A prototypic model of 3D morphometry used in the 3D measurement system for construction robots was designed and manufactured based on the component design concept. The 3D morphometry is used to acquire the data on filed topographical features around the construction machine in the site coordinate system by using the 3D measuring instrument mounted on the machine. The 3d coordinates of topographical information in the site coordinate system are created by following the steps: (1) Measure topographical features, using the 3D measuring instrument installed at the measurement reference point of the coordinate system that is set on the construction machine (hereinafter referred to as the machine body coordinate system); (2) Acquire the positional data on the measurement reference point being measured in the site coordinate system and on the axial rotation in the machine body coordinate system; and (3) The measured data in the machine coordinate system that was acquired through the $3 \mathrm{D}$ data measuring instrument is coordinate-converted into the site coordinate system in order to get the topographic data in the site coordinate system on the basis of the positional data on the measurement reference point in the site coordinate system and on the axial rotation data in the machine body coordinate system. Since the sets of data measured in steps (1) and (2) are independent of each other, the measuring instruments to be used can be simply handled as components. The instrument used in step (1) is termed the 3D data measurement component, while the one used in step (2) is called the machine position measurement component. As for the 3D data measurement component in step (1), two kinds of technologies (related to stereo display or "Stereovision" and laser scanning), which are based on the operational principles differing from each other, were embodied into two different components and put to experimental use.

\subsection{Machine position measurement component}

1) Outline

The machine position measurement component is the one that measures, in real time, the position/attitude/azimuth data (corresponding to the positional data on the reference measurement point and to the data on the axial rotation of the machine body coordinate system as described in step (2) above) on the machine that has the $3 \mathrm{D}$ data measuring instrument on it in the site coordinate system. Sets of acquired position/attitude/azimuth data on the machine are time-synchronized and, in response to the request from other measurement components, are sent to them to be used as information about coordinate system conversion.

2) Configuration of instruments

Each of multiple sets of data has to be measured with its own sensor. A GPS receiver is used to measure the machine position in the site coordinate system, while an optical fiber gyroscope unit is used to measure the inclinations (pitching and rolling angles) in the machine body coordinate system. A vector sensor is used to measure the azimuth angle (yawing angle).

A configuration of the machine position measurement component is given in Figure 4-1 and the component installed on the backhoe is shown in Figure 4-2. 


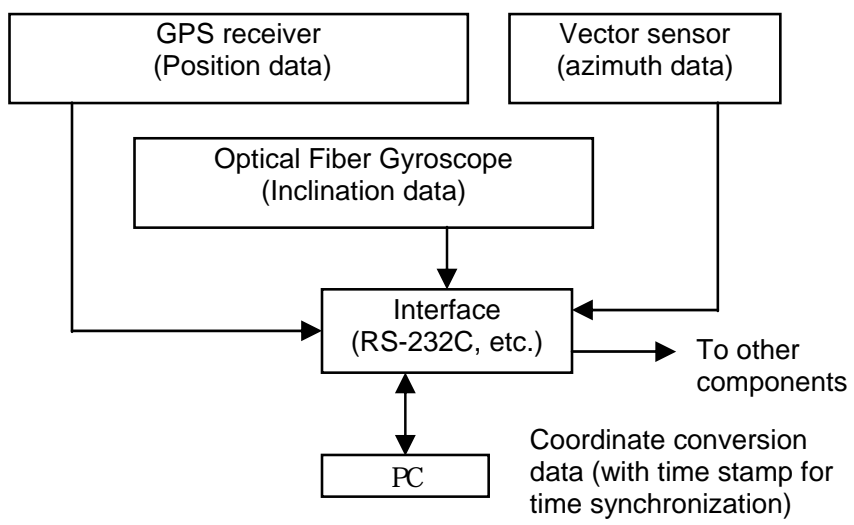

Figure 4-1 Configuration of Machine Position Measurement Component

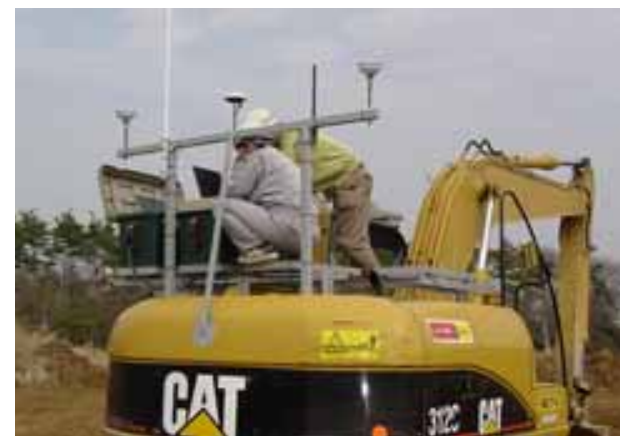

Figure 4-2 Machine Position Measurement Component on Excavator

3) Time synchronization process

Information to be used in the 3D data measurement component for coordinate system conversion is created by means of software installed in the PC as the machine position measurement component. A concept of time synchronization process for the data from multiple sensors is illustrated in Figure 4-3. As shown here, it is ordinarily considered that data outputs from the sensors are not synchronously timed partly because measurement frequency differs from one sensor to other. Hence the need for theoretically generating the data for ensuring synchronized timing.

For the synchronous process, it is necessary in the first place to find the latency required by each sensor. The data on the synchronous time is calculated by taking the latency into account. Proportional distribution process should be used to compute that data from the preceding as well as succeeding data. Thus, the resultant data is outputted as the synchronous time data, which is in principle given in response to the request from other components, such as the 3D data measurement components. It should be noted that there are particularly no master-slave relationships among the components concerned.

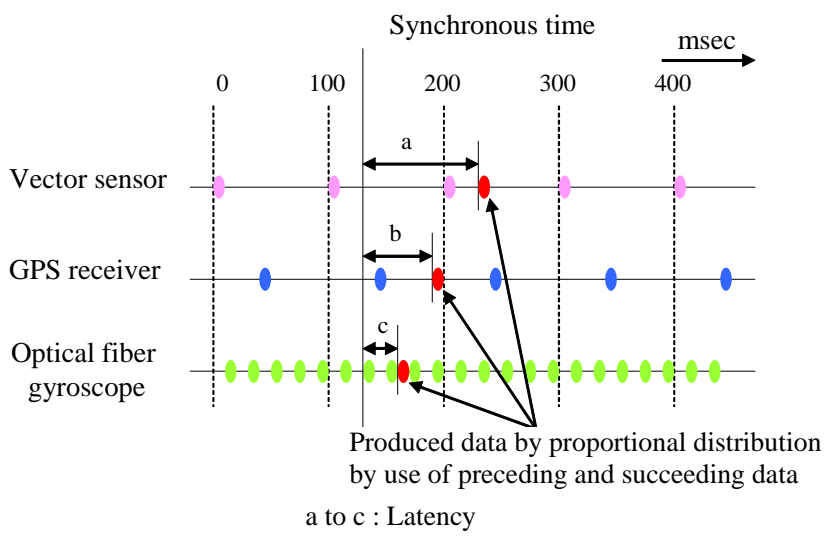

Figure 4-3 Time Synchronization Process

\subsection{D data measurement components}

1) Outline

The 3D data measurement component is designed to measure the topographical features in the machine body coordinate system with the 3D data measuring instruments mounted on the construction machine, and to convert the data so acquired into the topographical data in the site coordinate system using the coordinate conversion information provided by the machine position measurement component.

Taking full advantage of the component design concept, two measuring instruments that are based on totally different operational principles have been adopted, i.e., Stereovision and a 2D laser scanner. Prototypes of these two instruments to be incorporated in the two independent components have been manufactured and tested. Stereovision uses images gained from two digital cameras in order to acquire the distance information by means of triangulation techniques, thereby rendering the view covered by the cameras three-dimensional. On the other hand, using a rotating mirror that radiates a pulsed laser light used for range-finding application in sectorial fashion, the 2D laser scanner can present a 3D delineation of the range measured by it.

2) Configuration of Stereovision system component The Stereovision system component measures topographical features with Stereovision mounted on the construction machine and to covert the measurements into the topographical information in the site coordinate system.

A configuration of the Stereovision system component is given in Figure 4-4 and experimental use of the component is shown in Figure 4-5. 


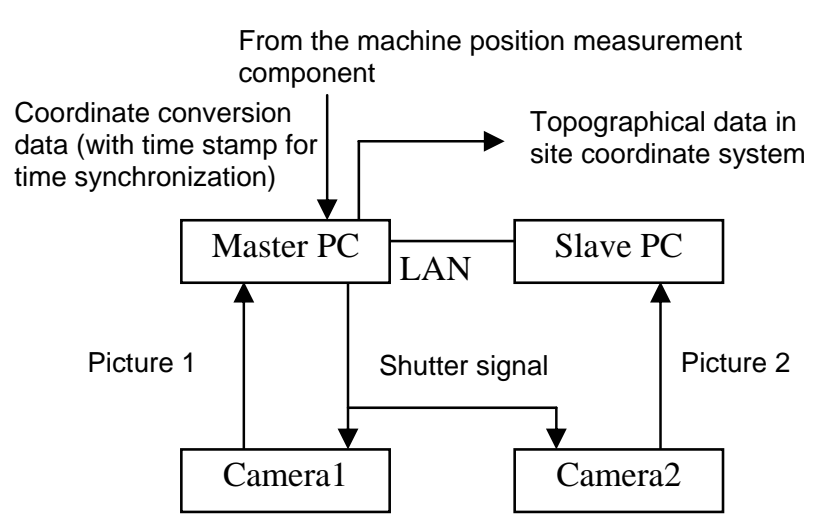

Figure 4-4 Configuration of Stereovision System Component

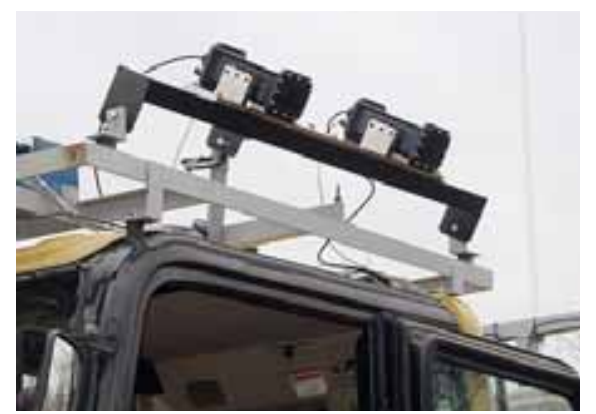

Figure 4-5 Stereovision System Component Mounted on Excavator

3) Configuration of laser scanner system component The laser scanner system component measures topographical features with the laser scanner system mounted on the construction machine and to convert the measurements into the topographical information in the site coordinate system.

A configuration of the laser scanner system component is given in Figure 4-6 and experimental use of the component is shown in Figure 4-7.

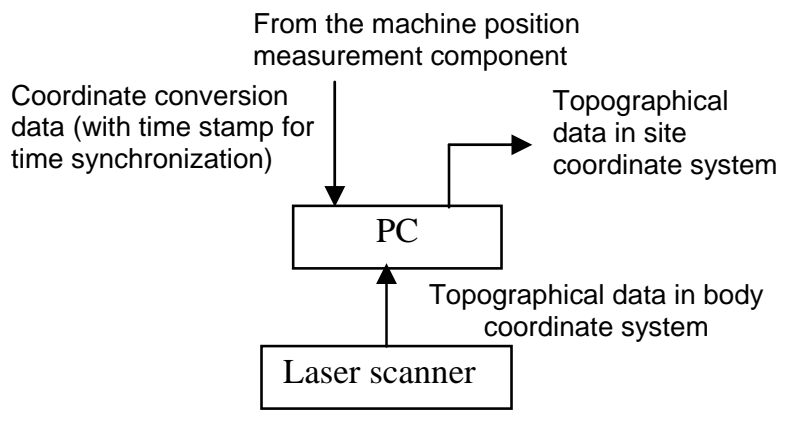

Figure 4-6 Configuration of Laser Scanner System Component

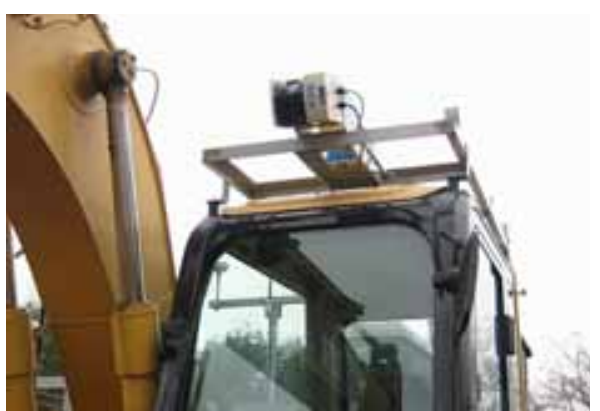

Figure 4-7 Laser Scanner System Component Mounted on Excavator

\section{Testing of Excavator Equipped with Components}

The machine position measurement component, Stereovision system component, and laser scanner system component were mounted on an excavator and the measurement and excavation capabilities were tested. The excavator is a hydraulic power shovel with a packet capacity of $0.5 \mathrm{~m}^{3}$. Targets of the experiment are clay ground for excavation and a plywood shield of $2.7 \mathrm{~m} \mathrm{x}$ $2.7 \mathrm{~m}$ for measurement that was inclined 45 degrees.

1) Findings

In the experiment, coordinates of the targets as the end products of the experiment were calculated. Evaluated were such factors as measurement accuracy, processing time, and measurement density as well as information exchange between components. A picture of the experiment is shown in Figure 5-1. The 3D data measurement technology based on the Stereovision and laser scanner system components that are called the Stereovision method and the laser scanner method, respectively. is discussed in relation to each evaluation item.

(1) Measurement accuracy (Requirement: $\pm 50 \mathrm{~mm}$ )

- Stereovision method

The maximum error tolerance is less than $\pm \mathrm{mm}$ when the shovel is at rest, but when it is swiveling, this requirement cannot be met.

- Laser scanner method

The standard deviation measured is $13 \mathrm{~mm}$ when the shovel is at rest, and $43 \mathrm{~mm}$ when it is swiveling. When it is swiveling over 30 degrees per second, the requirement cannot be met.

(2) Processing time (Requirement: less than 10 seconds)

- Stereovision method (Resolution: 3 million pixels)

Entire processing time is 21 seconds, which is broke down into 16 seconds for stereo processing and into5 seconds for coordinate conversion and other processing.

- Laser scanner method

Entire processing time is about $200 \mathrm{msec}$, including the sensor latency, synchronization 
processing, and successive coordination conversion.

(3) Measurement density (Requirement: A pitch between two adjacent points on the coordinates should be $10 \mathrm{~cm}$.)

- Stereovision method (Requirement: 3 million pixels)

A point-to-point pitch at a measurement distance of $10 \mathrm{~m}$ is $5 \mathrm{~mm}$ (maximum resolution). The measured mesh occupancy is $100 \%$ when the shovel is turning.

- Laser scanner method

Mesh occupancy is $88 \%$ when the shovel is swiveling 6 degrees per second.

Mesh occupancy is 50\% when the shovel is swiveling 20 degrees per second.

Note: Mesh capacity=Number of $10-\mathrm{cm}$ meshes, each having measurement points per unit area.

(4) Measurement range (Requirement: $10 \mathrm{~m} \mathrm{x} 10 \mathrm{~m}$ )

- Stereovision method (Resolution: 3 million pixels)

Trapezoid (Upper hem: 5,800 mm; Lower hem: 2,075 mm; Height: 4,650 mm)

- Laser scanner method

Circular (360 degrees) with a radius of $20 \mathrm{~m}$ or more

2) Discussion and tasks

The findings from the experiment indicate that the Stereovision method could be suitable for applications where topographic features within the limited area should be known in detail, while the laser scanner method could be preferred when such features are to be quickly grasped over a relatively wide range of area. Table 5-1 lists the results from comparative study on the two methods with respect to their requirements. As can be seen from the table, these two methods have features that might be complementary to each other. This probably implies two practices. One of them is to use both methods at the same time so that information from the one could be effectively used by the other, and vice versa. The other practice is to selectively use one of the two methods that would be more suitable for the specific target.

Tasks to be taken up for improvements in the Stereovision method are to increase the measurement accuracy when the shovel is swiveling; to shorten the processing time; and to expand the measurement range. An enhancement in the measurement accuracy will be made possible by analyzing the dynamic measurement characteristics. The processing time and measurement range will be improved through the review/update of the specifications concerned and the enhancement of PC capability. On the other hand, the laser scanner method should heighten the measurement density with an increasing number of scanners installed as well as with modified specifications. A task common to both methods is most likely to increase the measurement accuracy in the azimuth information (i.e., yawing angle) for the machine position measurement component when the shovel is turning over 30 degrees per second.

Figures 5-2 and 5-3 show the measured data derived from the Sterovision method and the laser scanner method, respectively. The results are displayed using a dedicated viewer software after coordinate conversion of the measurement data.

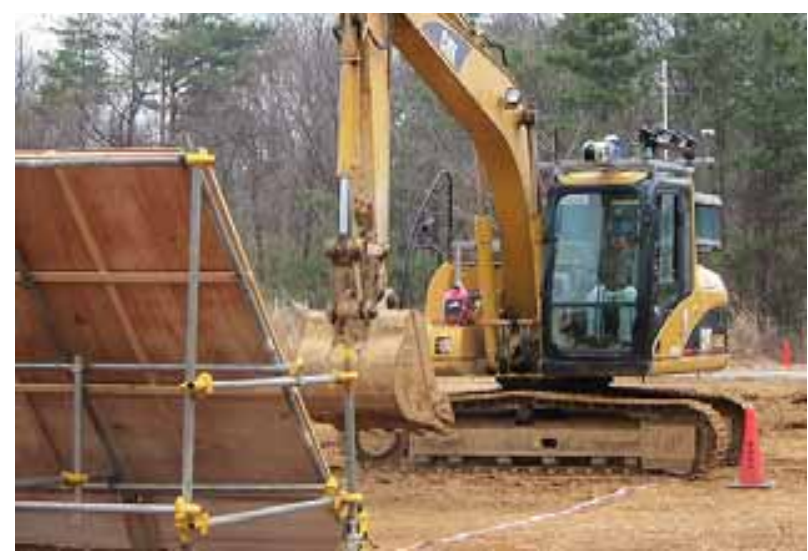

Figure 5-1 Measurement Experiment by the Excavator Equipped with All Components

Table 5-1 Features Compared Between the Methods

\begin{tabular}{|c|c|c|c|}
\hline & Requirements & Stereovision method & Laser scanner method \\
\hline Accuracy & $\pm 50 \mathrm{~mm}$ & & \\
\hline Processing time & $\begin{array}{l}\text { Less than } \\
10 \mathrm{sec}\end{array}$ & & (0) \\
\hline $\begin{array}{l}\text { Measurement } \\
\text { accuracy }\end{array}$ & 10-cm pitch & (0) & $\angle$ \\
\hline Measurement & $\begin{array}{c}\text { range } \\
10 \mathrm{~m} \times 10 \mathrm{~m}\end{array}$ & $\triangle$ & (0) \\
\hline
\end{tabular}




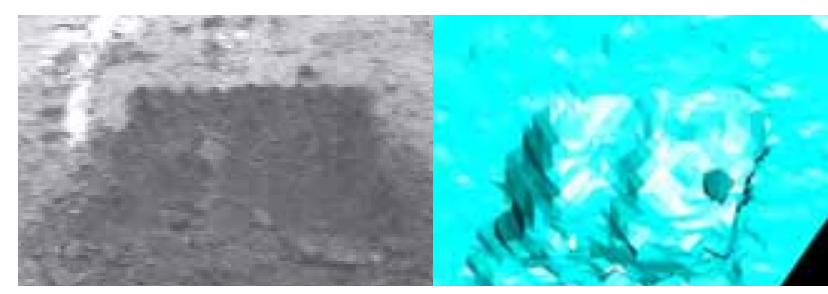

Figure 5-2 Measured Data trom Stereovision with Customized Viewer

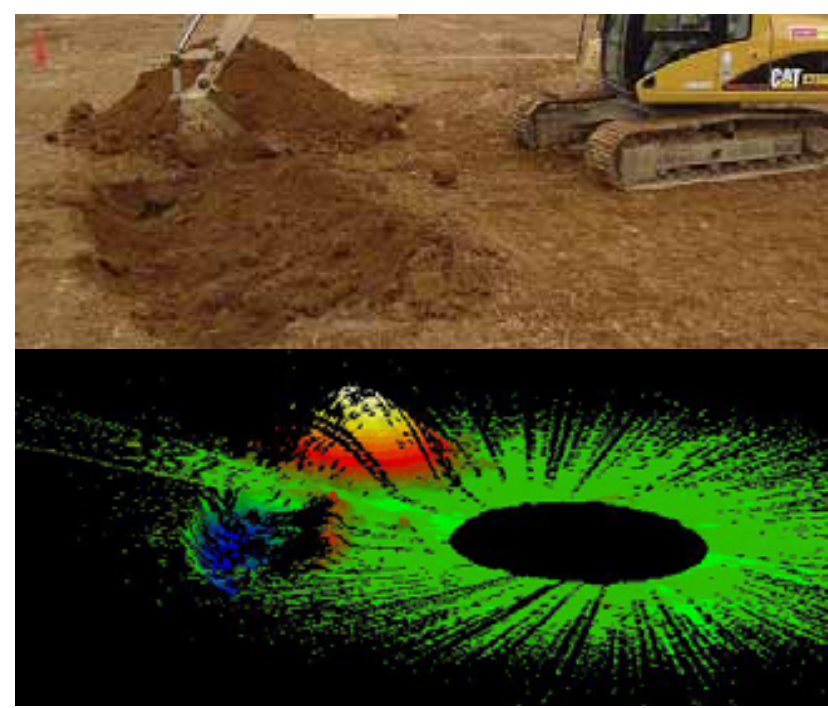

Figure 5-3 Measured Data from Laser Scanner with Customized Viewer

\section{Conclusion}

1) The prototype of 3D morphometry technology of the construction machine has been designed and put to experimental use based on the component design concept.

2) Two independent measurement components, which are built around measuring instruments having different features, have been designed and tested as prototypes.

3) The test indicates that, when identical construction machine is used, information obtained could be desirably shared between the two components through their selective or concurrent use depending on the objects to be measured.

\section{Acknowledgment:}

We wish to express our thanks to Assistant Professor T. Tsubouchi of Tsukuba University and many other people for giving their kind cooperation in our research.

\section{References:}

[1] Ishikawa, Ageishi, Yamamoto: "The Development of Shotcrete Robot for Sabo Works of Mr. Fuji”, 10th
Symposium on Construction Robotics in Japan, September 2004.

[2] John Cheesman, John Daniels: "UML Component Design” translated by Nagase and Knno, Pearson

Education In., May 2002. 